\title{
The Implementation of a Tobacco Use Registry in an Academic Family Practice
}

\author{
Dana Neutze, MD, PhD, Carol Ripley-Moffitt, MDiv, Mark Gwynne, DO, \\ and Adam O. Goldstein, MD, MPH
}

Purpose: Patients who use tobacco often are not provided evidence-based interventions because of barriers such as lack of time or expertise. Using a chronic disease model, we sought to improve delivery of care with an innovative decision support tool and a tobacco use registry.

Methods: We designed and implemented a decision support tool in an academic family medicine clinic. To assess barriers, we measured duration of visit and provider confidence (scale of $0-10$ ) in prescribing cessation medications before and after the introduction of the tool. We examined fidelity through daily counts of returned forms.

Results: No significant differences in mean office visit cycle times occurred for tobacco users $(64.7 \mathrm{vs}$ 63.1 minutes; $P=.90)$ or between tobacco users and nontobacco users $(63.1$ vs 62.5 minutes; $P=$ 1.00) before or after implementation of the decision support tool. Mean provider confidence in prescribing cessation medications increased significantly for nicotine inhalers $(4.8$ vs $6.4 ; P=.01)$, nicotine nasal spray $(3.9$ vs $5.5 ; P=.03)$ and combination nicotine replacement therapy $(5.5$ vs $6.2 ; P=$ $.05)$. Two years after implementation, $88 \%$ of forms were filled out and returned daily, and $>2200$ tobacco users have been entered into the registry.

Conclusions: The tobacco use decision support tool resulted in an increase in provider confidence in prescribing cessation medications without lengthening the duration of patients' visits, and the tool continues to be used routinely in the practice 2 years after introduction, indicating sustainability. The use of a tobacco use registry and decision support tool aids in standardizing care and overcoming barriers to cessation counseling. (J Am Board Fam Med 2015;28:214-221.)

Keywords: Chronic Disease, Counseling, Registries, Smoking Cessation, Substance Abuse

Recent work suggests that tobacco use should be viewed as a chronic illness, with a similar investment on the part of the clinician in long-term

This article was externally peer reviewed.

Submitted 10 April 2014; revised 8 October 2014; accepted 27 October 2014.

From the Department of Family Medicine, University of

North Carolina School of Medicine, Chapel Hill.

Funding: none.

Prior presentation: The work was presented at the Society of Teachers of Family Medicine Practice Improvement Conference, Greenville, SC (2012).

Conflict of interest: none declared.

Corresponding author: Dana Neutze, MD PhD, Department of Family Medicine, University of North Carolina School of Medicine, 590 Manning Drive, Chapel Hill, NC 27599 (E-mail: dana_neutze@med.unc.edu).

See Related Article on Page 205. treatment. ${ }^{1,2}$ Indeed, with the implementation of the Physician Quality Reporting System, physicians will not only report care for diseases such as diabetes and congestive heart failure, they also will be required to report screening and treatment for tobacco use. Therefore, using a registry and/or a clinical decision support tool to standardize patient care around tobacco use is a natural extension of treating chronic diseases.

To provide optimally effective tobacco use treatment in clinic settings, the United States Department of Health and Human Services recommends the "5 As": ask about tobacco use; advise cessation; assess willingness to quit; assist in a quit attempt; and arrange follow-up. ${ }^{3}$ A recent survey of health professionals found that while the great majority of primary care providers reported asking whether a patient smokes and advising quitting, significantly fewer providers reported assisting patients with 
quitting or arranging follow-up. ${ }^{4}$ Only two thirds of providers discussed medications or reported helping patients set a quit date, and only half reported referring patients to a cessation program or providing materials about quit lines. A Center for Disease Control and Prevention report looking at 96,232 outpatient visits of patients $\geq 18$ years old from 2005 to 2007 found even lower counseling rates, with documented evidence of counseling among patients using tobacco at $20.9 \%$ and cessation medication offers at $7.6 \% .^{5}$

Common barriers to addressing the 5 As on the part of providers include lack of time, patient lack of readiness to change, inadequate resources, language and culture barriers, patient nonadherence, and inadequate clinical skills (eg, motivational interviewing, problem solving, awareness of pharmacology) for counseling about cessation. ${ }^{6,7}$ Effective interventions need to address one or more of these barriers. Studies of the adoption of other clinical practice guidelines show that practitioner education alone does not lead to the successful adoption of clinical practice guidelines. ${ }^{8}$ Workflow-focused interventions, however, have been proven to increase successful adoption, even without concurrent provider education. ${ }^{9}$ One particularly effective workflow-focused intervention, a clinical decision support system, increased guideline-oriented care in $14 \%$ to $68 \%$ of encounters. ${ }^{10}$ Moreover, when this decision support tool is automatically integrated into clinician workflow, the implementation of guidelines is improved by up to $75 \% .{ }^{11}$

The current electronic health record (EHR) used at the University of North Carolina (UNC) records the first 3 "As" of tobacco status in the vital signs. Medical assistants ask whether the patient uses tobacco, advise the patient to quit, and assess whether the patient has plans for, or is interested in, quitting tobacco use. Asking about tobacco use as part of the vital sign assessment has significantly increased counseling to assist patient quit attempts. ${ }^{12}$ The UNC Family Medicine Center (FMC) currently has 17,200 empanelled patients, accounting for 58,000 visits per year. Because of our high rates of tobacco use documentation, we know that of this number, $>3000$ patients currently smoke or use other tobacco products. To improve rates of addressing the fourth "A"- -assisting with a quit attempt-in this large patient population, and in preparation for patient-centered medical home certification, an FMC quality improvement team de- veloped a tobacco use clinical decision support tool. Although some EHRs have built-in clinical support tools around tobacco use, as far as we are aware this is the first report of a comprehensive, evidencebased tobacco decision tool modeled after other successful chronic disease registry models. This article addresses the design and implementation of the decision support tool and accompanying registry, examining their effect on overcoming common provider barriers, including lack of time and clinical skills related to adequate tobacco use treatment.

\section{Methods}

\section{Designing the Decision Support Tool}

The tobacco use decision support tool, designed by FMC physicians and an FMC tobacco treatment specialist, was modeled on existing chronic disease decision support tools (ie, congestive heart failure, diabetes, coronary heart disease) already used within the clinic. The individualized form, with the patient's biometrics, consists of 3 sections (see Appendix). The first contains prepopulated data, listing tobacco use status, patient-reported importance of quitting score, and blood pressure measurements from the previous 5 visits. The second section lists medical assistant responsibilities: recording current tobacco use status, number of cigarettes or amount of other tobacco used per day; patient's current self-reported importance score for quitting and interest in quitting in the next 30 days; referrals offered (ie, the onsite FMC Nicotine Dependence Program [NDP] and QuitlineNC); and educational materials given. Additional items in the second section include the 2-item Patient Health Questionnaire depression screen, documentation of pneumococcal vaccination administration, and, for men $>65$ years old, guideline-recommended aortic aneurysm screening. ${ }^{13}$ The third section contains a list of recommended services to prompt clinicians, as well as a section for recording a quit date and any treatments offered (ie, medication or counseling). The recommended services box lists appropriate care steps in red type. Once a particular step is marked as completed on the form, it no longer appears on subsequent printings. A reminder to code for counseling remains on the form in green type indefinitely. An accompanying reference sheet lists initial doses of cessation medications.

We piloted the tobacco use decision support tool with 1 of the 4 FMC clinical teams before 
disseminating it to the entire practice; medical assistants and clinicians gave feedback on the ease of use and workflow. We revised the form several times based on these rapid-cycle Plan Do Study Act trials. Once the tool was ready to be implemented throughout the clinic, we held a 15-minute didactic session during a regular monthly meeting for the entire clinic. All providers and medical assistants were taught how to use the tool and were able to ask questions. The final version of the decision support tool with the accompanying pharmacology reference is shown in the Appendix.

A subgroup of patients were initially entered into the new tobacco use registry database. After it was determined that data were being entered and the system could support more patients, all patients who met the criteria (ie, positive for current tobacco use on vital signs within the past year) were entered into the registry. New patients continued to be entered into the registry during the weekly EHR system update.

The decision support tool forms were printed out each evening based on the following day's schedule for continuity patients who currently use tobacco. Forms were turned in each day, and updates were recorded into the registry database by a staff member. Materials offered to patients included 1 of 2 self-management tools based on patient's readiness to quit and a brochure describing the services of the NDP. Fax referral forms for QuitlineNC were prepopulated with the FMC clinic name and contact information and placed in every examination room. Medical assistants were instructed to offer the patient a referral to QuitlineNC and to fill out the form if the patient agreed. Medical assistants were also instructed to ask patients whether they were willing to attend the NDP and make the necessary referral. Physicians and nurse practitioners also were able to make both of these referrals if necessary after further discussion with the patient.

\section{Implementation Barriers and Fidelity}

To address concerns about common barriers, namely, time and provider knowledge, we examined the duration of patient visits and provider confidence in prescribing cessation medications. To assess the fidelity of using the tobacco use decision support tool, we measured the return rate and documentation rate 2 years after implementation.

\section{Office Visit Cycle Times}

We recorded office visit cycle times, defined as the total time from a patient's entry into the building until they exit, for every patient visit in the Patient Tracker module of the clinic's scheduling system. We compared the mean duration of an office visit between tobacco users and nontobacco users using Student $t$ tests for both before (May 2011 through October 2011) and after (January 2011 through June 2012) implementing the registry. In addition, we compared the mean duration of a visit for tobacco users before and after the tool using the Student $t$ test. We excluded November and December 2011 from the analysis because only a portion of tobacco users were enrolled in the registry at that time.

\section{Provider Confidence}

We e-mailed an invitation to participate in an online survey to all 60 providers ( 24 residents and 36 attendings) in the clinic before and after implementation of the tobacco use decision support tool to assess their prescribing behavior and confidence in prescribing cessation medications. We compared confidence scores (using a scale of 0 to 10: $0=$ not at all, $10=$ extremely confident) for providers who answered surveys both before and after implementation of the tool with both 2-way analysis of variance and paired $t$ tests. The UNC Institutional Review Board approved this survey.

\section{Continued Usage}

We tracked the total number of both tobacco and other chronic disease forms printed, returned, initialed, and with documented changes for 2 months (September and October 2013), almost 2 years after registry and decision support tool initiation, to determine continued fidelity to the intervention. We compared the percentage of returned forms for patients with tobacco use versus those with other chronic diseases using the Student $t$ test. We calculated odds ratios for the likelihood of medical assistants initialing the form compared with providers and for medical assistants noting new information compared with providers.

\section{Results}

The decision support tool was rolled out across all 4 clinic teams in November 2011 for patients already in the other chronic disease registries. In 
December 2011 all patients with a current tobacco use vital sign were added into the registry on a rolling basis.

\section{Visit Times}

There was no difference in the mean duration of the office visit cycle times between tobacco users and nontobacco users before or after implementation of the decision support tool (64.7 vs 62.79 minutes $[P=1.00]$ and 63.1 vs 62.5 minutes $[P=$ 1.00], respectively). Nor was there a difference in the mean total visit time for tobacco users before and after the tool (64.7 vs 62.7 minutes; $P=.86$ ).

\section{Provider Confidence}

In total, 30 and 42 surveys were completed before and after implementation of the tool, respectively (response rate of $50 \%$ and $70 \%$, respectively). For both surveys $52 \%$ of respondents were residents. Seventeen individuals completed both pre- and postregistry surveys (Table 1 ). There was a significant increase in the mean confidence of prescribing certain medications, including nicotine inhalers (4.8 vs $6.4 ; P=.01$ ), nicotine nasal spray ( 3.9 vs 5.5 ; $P=.03)$, and combination nicotine replacement therapy (5.5 vs $6.2 ; P=.05$ ), after the implementation of the tool and accompanying pharmacology reference sheet. There was a trend toward an increase in confidence when prescribing nicotine lozenges (5.7 vs $7.2 ; P=.06$ ).

\section{Continued Usage}

Rates of return and actions taken on the forms are listed in Table 2. Over the 2-month period, the daily mean rate of tobacco use forms returned for patients seen was $88 \%$ (range, $57-100 \%$ ). This was not significantly different from the return of the other chronic disease forms during the same time period, which was $85 \%$ (range, $47-100 \%$; $P=.33$ ). Over $80 \%$ of returned forms were initialed by either a medical assistant and/or a provider to verify that he or she had reviewed the form. There was no significant difference in the number of forms signed by medical assistants versus providers (odds ratio [OR], 1.2; 95\% confidence interval [CI], 0.97-1.50). Almost three fourths of returned forms showed an update (ie, cigarettes per day, referral to QuitlineNC, counseling offered, medication offered). Medical assistants were significantly more likely than providers to write new information on the form (OR,
Table 1. Confidence Levels Prescribing Cessation Medications Before and After Implementation of the Decision Support Tool

\begin{tabular}{|c|c|c|c|}
\hline \multirow[b]{2}{*}{$\begin{array}{l}\text { Tobacco } \\
\text { Cessation } \\
\text { Medication } \\
\text { Prescribed, by } \\
\text { Physician }\end{array}$} & \multicolumn{2}{|c|}{ Confidence Levels } & \multirow[b]{2}{*}{$\begin{array}{c}P \\
\text { Value* }\end{array}$} \\
\hline & $\begin{array}{c}\text { Before } \\
\text { Implementation } \\
\text { of the Decision } \\
\text { Support Tool }\end{array}$ & $\begin{array}{l}\text { After Before } \\
\text { Implementation } \\
\text { of the Decision } \\
\text { Support Tool }\end{array}$ & \\
\hline Nicotine gum & & & .19 \\
\hline Resident & 7.0 & 7.8 & \\
\hline Faculty & 7.4 & 8.8 & \\
\hline Nicotine patch & & & .34 \\
\hline Resident & 7.0 & 8.2 & \\
\hline Faculty & 8.7 & 8.8 & \\
\hline Nicotine lozenges & & & .06 \\
\hline Resident & 5.8 & 7.4 & \\
\hline Faculty & 5.7 & 7.7 & \\
\hline Nicotine inhaler & & & .01 \\
\hline Resident & 4.9 & 5.9 & \\
\hline Faculty & 4.8 & 7.2 & \\
\hline Nicotine spray & & & .03 \\
\hline Resident & 4.0 & 4.6 & \\
\hline Faculty & 3.9 & 6.8 & \\
\hline $\begin{array}{c}\text { Combination } \\
\text { NRT }\end{array}$ & & & .05 \\
\hline Resident & 4.8 & 6.3 & \\
\hline Faculty & 5.4 & 6.7 & \\
\hline Wellbutrin & & & 1.00 \\
\hline Resident & 5.7 & 6.3 & \\
\hline Faculty & 9.4 & 6.7 & \\
\hline Chantix & & & .76 \\
\hline Resident & 5.0 & 5.7 & \\
\hline Faculty & 8.8 & 8.7 & \\
\hline
\end{tabular}

*Paired $t$ test by cessation method, resident and faculty combined.

NRT, nicotine replacement therapy.

3.26; $95 \%$ CI, 2.62-4.04). Within the first 2 years, $>2200$ patients in the practice were entered into the tobacco use registry.

\section{Discussion}

The FMC has a history of using chronic disease registries and clinical support tools to help in the management of chronic diseases, including diabetes, congestive heart failure, and coronary artery disease. Therefore, we took a similar approach to coordinate care of patients who use tobacco. We designed the tobacco use decision support tool to increase offers of evidence-based tobacco use treatment to patients. These included prompts to refer to the onsite NDP and 
Table 2. Mean Daily Rates of Registry Forms Returned, Initialed, and Updated, September Through October 2013

\begin{tabular}{lcc}
\hline & \multicolumn{2}{c}{ Daily Rate (\%) } \\
\cline { 2 - 3 } & Mean & Range \\
\hline Tobacco use registry forms returned & 88 & $57-100$ \\
$\begin{array}{l}\text { Other chronic disease registry forms } \\
\text { returned }\end{array}$ & 85 & $47-100$ \\
$\begin{array}{l}\text { Tobacco use registry initialed by medical } \\
\quad \text { assistant and/or provider }\end{array}$ & 82 & $58-100$ \\
\begin{tabular}{l} 
Tobacco use registry updated or changed \\
\hline
\end{tabular} & 73 & $40-100$ \\
\hline
\end{tabular}

the state telephone quit line, as well as to recommend counseling and medications. (For outcomes data for these measures, see the companion article in this issue. ${ }^{14}$ )

The decision support tool has become a routine part of the clinical workflow. Even after 2 years of use, the great majority of our clinicians still use the form on a daily basis. Not only are the majority of forms initialed and returned, three-quarters contain new information that is subsequently entered into the registry. It is reassuring to note that both medical assistants and providers are continuing to use the form.

The use of a clinical support tool helped to address some of the common physician barriers to care of patients who use tobacco: lack of time and clinical skills in tobacco use treatment. Using the tool did not lengthen the patients' office visit cycle times; overall visit time was the same before and after the intervention. We recognize that this measure includes more than just the time spent with the provider, but we were unable to accurately record the shorter interval. By having referrals, resources, and a pharmacologic reference sheet readily available, less time is needed to coordinate these efforts. Furthermore, we found an increase in confidence in using some of the cessation medications after implementation of the registry. It is hypothesized that the pharmacologic reference sheet distributed with all forms accounted for an increase in confidence scores regarding medication use, although additional training around the use of the decision support tool may have contributed to increased confidence. The biggest changes in confidence occurred with less commonly used medications, suggesting that some residents and faculty were perhaps previously unfamiliar with these evidence-based medications. Outcomes reported in the companion ar- ticle, ${ }^{14}$ based on a chart review, support the hypothesis that providers actually increased the number of cessation prescriptions provided to patients.

Several limitations existed in this process. Initially, push-back from medical assistants and providers occurred because of concerns about having an additional chronic disease form to complete during a patient visit (in addition to 3 others), creating a sense of burden or overload. Yet we included those most affected by the implementation, that is, providers and medical assistants, in the Plan Do Study Act cycles to create buy-in; we communicated changes during rapid cycle trials; and we reduced the form's complexity-all essential features to positively affect long-term change. We also needed to coordinate all self-management tools and QuitlineNC fax referrals on 4 different teams, using a standardized approach throughout the clinic. Despite these obstacles, the tobacco use decision support tool is now a regular part of clinic workflow.

A final observation is that our tobacco use decision support tool, like all our practice disease tools, was built and remains housed outside of our practice's EHR. This requires dedicated staff time to enter the results of each visit. In our practice this role is performed by a nonclinical chronic disease manager, but it could also be done by any available medical assistant. As health care moves toward a new era of EHRs, decision support tools like the one outlined in this article should be fully integrated into the EHR, enabling documentation and coordination of services at office visits, with individual provider feedback and without the need for an external tool.

The associated tobacco use registry has the ability not only to longitudinally track the care a patient receives but also to query the registry to facilitate population health management. The full capability of this feature, such as ensuring that all our patients with tobacco use receive a pneumococcal vaccination, is the next intervention for our team. We also will be incorporating lessons learned from this tobacco use registry into the new EHR being adopted by our health care system, tracking long-term outcomes, including quit rates, and exploring the feasibility of adopting this tool in nonacademic settings.

The authors thank Todd Meath and Ashley Packett for help with data collection and Jennifer Greyber for editorial assistance with manuscript preparation. The Health-e-NC initiative of the 
University Cancer Research Fund at the University of North Carolina contributed financial support for manuscript preparation.

\section{References}

1. Hudson NL, Mannino DM. Tobacco use: a chronic illness? J Community Health 2010;35:549-53.

2. Steinberg MB, Schmelzer AC, Lin PN, Garcia G. Smoking as a chronic disease. Curr Cardio Risk Rep 2010;4:413-20.

3. 2008 PHS Guideline Update Panel, Liaisons, and Staff. Treating tobacco use and dependence: 2008 update U.S. Public Health Service Clinical Practice Guideline executive summary. Respir Care 2008;53: 1217-22.

4. Tong EK, Strouse R, Hall J, Kovac M, Schroeder SA. National survey of U.S. health professionals' smoking prevalence, cessation practices, and beliefs. Nicotine Tob Res 2010;12:724-33.

5. Jamal A, Dube SR, Malarcher AM, Shaw L, Engstrom MC; Centers for Disease Control and Prevention (CDC). Tobacco use screening and counseling during physician office visits among adults- $\mathrm{Na}$ tional Ambulatory Med Care Survey and National Health Interview Survey, United States, 2005-2009. MMWR Morb Mortal Wkly Rep 2012;61:38-45.

6. Blumenthal DS. Barriers to the provision of smoking cessation services reported by clinicians in underserved communities. J Am Board Fam Med 2007;20: 272-9.
7. Caplan L, Stout C, Blumenthal DS. Training physicians to do office-based smoking cessation increases adherence to PHS guidelines. J Community Health 2011;36:238-43.

8. Davis D, Taylor-Vaisey A. Translating guidelines into practice: a systematic review of theoretic concepts, practical experience and research evidence in the adoption of clinical practice guidelines. Can Med Assoc J 1997;157:408-16.

9. Flanagan ME, Ramanujam R, Doebbeling BN. The effect of provider- and workflow-focused strategies for guideline implementation on provider acceptance. Implement Sci 2009;4:71.

10. Scott I. What are the most effective strategies for improving quality and safety of health care? Intern Med J 2009;39:389-400.

11. Kawamoto K, Houlihan CA, Balas EA, Lobach DF. Improving clinical practice using clinical decision support systems: a systematic review of trials to identify features critical to success. BMJ 2005; 330:765-68.

12. McCullough A, Fisher M, Goldstein AO, Kramer KD, Ripley-Moffitt C. Smoking as a vital sign: prompts to ask and assess increase cessation counseling. J Am Board Fam Med 2009;22:625-32.

13. U.S. Preventive Services Task Force. Screening for abdominal aortic aneurysm: recommendation statement. Ann Intern Med 2005;142:198-202.

14. Ripley-Moffitt C, Neutze D, Gwynne M, Goldstein AO. Patient care outcomes of a tobacco use registry in an Academic Family Practice. J Am Board Fam Med 2015;28:205-13. 
Appendix

Registry Decision Support Tool (Front and Back)

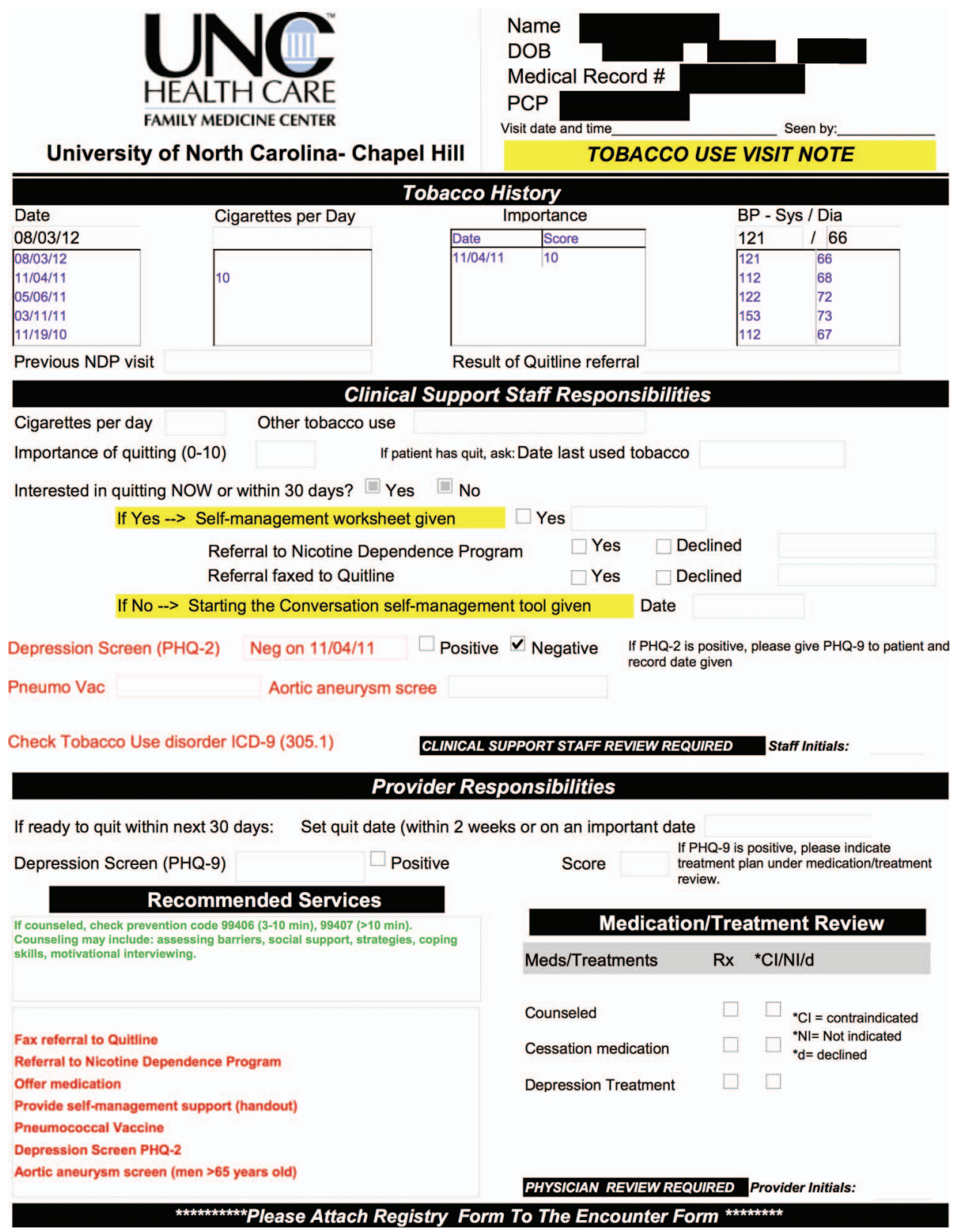


20 second example "Quitting all tobacco use is one of the most important things you can do for your health (to increase your energy, decrease SOB, improve your breathing, etc.). I understand that quitting can be difficult and I am here to support you. We have effective resources such as medication, a trained counselor, a support group, and the Quitline to help you succeed. Would you be willing to talk about your next steps for becoming tobacco free?

Social support (e.g., offering encouragement, identifying sources of support)

Strategies (e.g., tobacco free car/home, separating cigarette and coffee/alcohol)

Coping skills (e.g., deep breathing, reframing negative self-talk)

For those not currently willing:

Used motivational interviewing (e.g., What do you enjoy about smoking? What makes it difficult to think about quitting? Tell me about any times you have tried to quit.)

\section{Medications}

NC Medicaid covers OTC patch, gum, and lozenge with prescription.

NICOTINE REPLACEMENT (Combination patch with gum, lozenges more effective than patch alone.

\section{$\leq 1 / 2$ pack per day}

- Gum 2mg gum q 1-2 hrs PRN nicotine withdrawal

- Lonzenges $2 \mathrm{mg}$ gum q 1-2 hrs PRN nicotine withdrawal

$1 / 2$ - 1 pack per day

- Nicoderm $14 \mathrm{mg}$ transderm daily $x$ until 2 weeks without significant symptoms then

$7 \mathrm{mg}$ transderm daily $x$ until 2 weeks without significant symptoms

- Gum 2mg gum q 1-2 hrs PRN nicotine withdrawal

- Lonzenges 2mg gum q 1-2 hrs PRN nicotine withdrawal

\section{$>1$ pack per day}

- Nicoderm $21 \mathrm{mg}$ transderm daily $x$ until 2 weeks without significant symptoms then

$14 \mathrm{mg}$ transderm daily $x$ until 2 weeks without significant symptoms

$7 \mathrm{mg}$ transderm daily $x$ until 2 weeks without significant symptoms

- Gum 4mg gum q 1-2 hrs PRN nicotine withdrawal

- Lonzenges 4mg gum q 1-2 hrs PRN nicotine withdrawal

Nasal spray and inhalors also acceptable therapy

BUPROPRION SR 150mg (Wellbutrin) (may be used with nicotine replacement therapy)

Start 1-2 weeks BEFORE quit date

Days 1-3: 1 tab $Q$ day

Days 4-end: 1 tab BID

Days 4-end: 1 tab BID

VARENICLINE (Chantix)

Start 1 week BEFORE quit date

Starting month pak

Continuing month pak

Use 3-6 months 